\title{
ON THE STABILITY OF THE COHOMOLOGY OF COMPLEX STRUCTURES
}

\author{
BY \\ TAPIO KLEMOLA
}

\begin{abstract}
Let $\mathscr{V} \stackrel{\pi}{\rightarrow} M$ be a differentiable family of compact complex manifolds $V_{t}=\pi^{-1}(t)$ on $M=\left\{t \in R^{m}|| t \mid<1\right\}, \mathscr{B} \rightarrow \mathscr{V}$ a differentiable family of holomorphic vector bundles $B_{t} \rightarrow V_{t}, t \in M$. In this paper we study conditions for the cohomology groups $H \frac{r_{\partial_{t}} s}{s}\left(B_{t}\right)$ to be constant in a neighborhood of $0 \in M$.
\end{abstract}

1. Introduction. In the paper [4] the question of the constancy of the dimension of the groups $H_{\bar{\partial}_{t}}^{r, s}\left(B_{t}\right)$ of bundles $B_{t} \rightarrow V_{t}, t \in M$, of a differentiable family $\mathscr{B} \rightarrow \mathscr{V} \rightarrow M$ was studied. The notion of transportable forms was introduced, and those of extendible and co-extendible forms (see Definition 2.1 below). It was shown in [4] that $\operatorname{dim} H_{\partial_{t}}^{r, s}\left(B_{t}\right)$ is locally constant at a point $t_{0} \in M$ if and only if all harmonic forms $\gamma \in \boldsymbol{H}^{r, s}\left(B_{t_{0}}\right)$ are transportable. This was shown to be equivalent with the hypothesis that all harmonic forms of the given type at $t=t_{0}$ are extendible and co-extendible (Theorem 2.1 below).

The purpose of this paper is to deduce conditions for all harmonic forms of a given bidegree at $t=t_{0}$ to be extendible in the given family. This will automatically give conditions for co-extendibility, and thus for the constancy of $\operatorname{dim} H_{\partial t}^{r, s}\left(B_{t}\right)$ in a neighborhood of $t_{0} \in M$. Applications and a further analysis of the conditions (which can be considered as a statement of the vanishing of certain obstructions) are planned to be given in a subsequent paper.

In $\$ 2$ some notations are introduced and the basic result of [4] is recalled. For further details see [4] and, for a general background, [5] and [6]. The trivial extension of the harmonic theory of forms with values in the holomorphic tangent bundle $T_{0}^{\prime}$ of $V_{0}$ to one for forms with values in $\mathscr{T}_{0}=T_{0}^{\prime} \oplus T_{0}^{\prime \prime}$ is discussed in $\S 3$. The structural forms $\varphi_{t}$ and $\xi_{t}$ which determine (each) the complex structure on $V_{t}$, are discussed in $\$ 4$. $\S 5$ gives a development of the form $\xi_{t}$, and in $\S 6$ the differential equations satisfied by an extension (resp. a co-extension) of a harmonic form are considered. The differential equation for an extension is seen to be equivalent with an "integral equation" with an "initial value" which is a closed form, and an integrability condition. A solution for the integral equation in the form of a converging series is obtained in $\S 7$, and the integrability condition is discussed in $\S 8$. Sufficient conditions for $\operatorname{dim} H_{\bar{\partial}_{t}}^{r, s}\left(B_{t}\right)$ to be locally constant are derived in $\S 9$, and

Received by the editors March 3, 1970.

AMS 1970 subject classifications. Primary 32G05; Secondary 53C55, 32C10, 32C35.

Key words and phrases. Complex manifolds, deformation.

Copyright (C) 1971, American Mathematical Society 
in the last section conditions which are necessary in the differentiable case but also sufficient in the analytic case are obtained.

In [2], Ph. Griffiths presents an abstract theory of extendible forms. The idea of solving the corresponding differential equation through an integral equation obtained from the harmonic theory is used there, as well as in the paper [7] of M. Kuranishi, but there the equation is one for the structural form $\varphi$ and nonlinear.

2. Preliminaries. Let $\mathscr{V} \stackrel{\pi}{\rightarrow} M$ be a differentiable family of compact complex manifolds over $M=\left\{t \in \boldsymbol{R}^{m}|| t \mid<\varepsilon\right\}$ where $\varepsilon$ is a positive real number, and $\mathscr{B} \stackrel{x}{\rightarrow} \mathscr{V} \stackrel{\pi}{\rightarrow} M$ a differentiable family of holomorphic vector bundles (see Definitions 1,2 in [6, p. 58]). Let $L^{r, s}$ be the vector space of differentiable sections of scalar forms of type $(r, s)$ on $V_{t}=\pi^{-1}(t)$. Let $T_{t}^{\prime} \oplus T_{t}^{\prime \prime}$ be the decomposition of the complexified tangent bundle of $V_{t}$ into the direct sum of the holomorphic tangent bundle $T_{t}^{\prime}$ and its conjugate bundle $T_{t}^{\prime \prime}$, and $\mathscr{F}_{t}$ the dual bundle of $T_{t}^{\prime}$. Then the vector space $L_{t}^{r, s}$ is the space of sections of the bundle

$$
\mathscr{F}_{t}(r, s)=\left(\bigwedge^{r} \mathscr{F}_{t}\right) \wedge\left(\bigwedge^{s} \overline{\mathscr{F}}_{t}\right)
$$

Denote by $L^{r, s}\left(B_{t}\right)$ the vector space of differentiable sections of $B_{t} \otimes \mathscr{F}_{t}(r, s)$, where $B_{t}=\chi^{-1}\left(V_{t}\right)$. Defining the operator

$$
\bar{\partial}_{t}: L^{r, s}\left(B_{t}\right) \rightarrow L^{r, s+1}\left(B_{t}\right)
$$

as in $\left[6\right.$, p. 61], and denoting by $Z_{\hat{\partial}_{t}}^{r, s}$ the respective kernel we set

$$
H_{\bar{\partial}_{t}}^{r, s}\left(B_{t}\right)=Z_{\bar{\partial}_{t}}^{r, s}\left(B_{t}\right) / \vec{\partial}_{t} L^{r, s-1}\left(B_{t}\right) .
$$

The case of scalar forms is obtained by setting $B_{t}=C \times V_{t}$. For $B_{t}=T_{t}^{\prime}$ we set $\bar{\partial}_{t}=D_{t}^{\prime}$. As $\mathscr{V}=V_{0} \times M$ for the differentiable structure, we can identify $V_{t}$ and $V_{0}$ as differentiable manifolds, hence also their complexified tangent bundles $\mathscr{T}_{t}$ and $\mathscr{T}_{0}=\mathscr{T}$.

Let $P_{t}$ and $Q_{t}$ be the projections corresponding to the direct sum $\mathscr{T}=T_{t}^{\prime} \oplus T_{t}^{\prime \prime}$, and let $\psi_{k}, \psi_{r, s}, \psi_{k}^{\prime}, \psi_{k}^{\prime \prime}, \psi_{r, s}^{\prime}$, and $\psi_{r, s}^{\prime \prime}$ denote the sections of the bundle $\mathscr{T} \otimes \sum_{r+s=k} \mathscr{F}(r, s), \mathscr{T} \otimes \mathscr{F}(r, s), T_{0}^{\prime} \otimes \sum_{r+s=k} \mathscr{F}(r, s), T_{0}^{\prime \prime} \otimes \sum_{r+s=k} \mathscr{F}(r, s)$, $T_{0}^{\prime} \otimes \mathscr{F}(r, s)$, and $T_{0}^{\prime \prime} \otimes \mathscr{F}(r, s)$, respectively.

Suppose there is given a hermitian metric on $V_{t}$ and on $B_{t}$, each depending differentiably on $t$. We can then introduce the inner product $(\alpha, \beta)_{t}$ for $\alpha, \beta \in L^{r, s}\left(B_{t}\right)$ with the norm $|\alpha|_{t}=(\alpha, \alpha)_{t}^{1 / 2}$ (see $\S 2$ in [4]). Letting $\vartheta_{t}$ be the adjoint of $\bar{\partial}_{t}$ with respect to this inner product, we get the family $\left\{\square_{t}\right\}_{t \in M}$ of strongly elliptic formally selfadjoint linear differential operators $\square_{t}=\vartheta_{t} \bar{\partial}_{t}+\bar{\partial}_{t} \vartheta_{t}$ acting on the spaces $L^{r, s}\left(B_{t}\right)$. If $\boldsymbol{H}^{r, s}\left(B_{t}\right)$ denotes the space of harmonic forms of type $(r, s)$ with values in $B_{t}$, or the kernel of $\square_{t}$, we have a canonical isomorphism

$$
\boldsymbol{H}^{r, s}\left(B_{t}\right)=H_{\partial t}^{r, s}\left(B_{t}\right)
$$

induced by the inclusion $\boldsymbol{H}^{r, s}\left(B_{t}\right) \subset Z^{r, s}\left(B_{t}\right)$.

In the spaces $L^{r, s}\left(B_{t}\right)$ we will also use the Sobolev norms \|\|$_{k}$ (see $\left.\S 2,[4]\right)$. 
We shall utilize the bracket operation $[L, M]$ for $\mathscr{T}$-valued forms $L, M$ as defined in $[1$, p. 35$]$.

In view of Lemma 1 of $[6$, p. 49] we may assume that, for the differentiable structure, $\mathscr{B}=B \times M$ where $B=B_{0}$ is a bundle over $V_{0}$. Hence, for instance, $L^{r, s}\left(B_{t}\right)$ can be considered as a subspace of

$$
L^{r+s}(B)=L^{r+s}\left(B_{0}\right)=\sum_{p+q=r+s} L^{p, q}\left(B_{0}\right)
$$

Definition 2.1. A $\bar{\partial}_{0}$-closed form $\gamma \in L^{r, s}\left(B_{0}\right)$ is extendible at $0 \in M$ if there is a neighborhood $U$ of 0 and a family $\left\{\eta_{t}\right\}_{t \in U}$ of forms $\eta_{t} \in L^{r, s}\left(B_{t}\right)$ depending differentiably on $t$ and such that $\bar{\partial}_{t} \eta_{t}=0$ and $\eta_{0}=\gamma$. The form $\eta_{t}$ depending on the parameter $t$ is called an extension of $\gamma$. Similarly a $\vartheta_{t}$-closed form $\gamma \in L^{r, s}\left(B_{0}\right)$ is co-extendible at $0 \in M$ if there is a neighborhood $U$ of 0 and a family $\left\{\sigma_{t}\right\}_{t \in U}$ of forms $\sigma_{t} \in L^{r, s}\left(B_{t}\right)$ depending differentiably on $t$ such that $\vartheta_{t} \sigma_{t}=0$ and that $\sigma_{0}=\gamma$. The form $\sigma_{t}$ depending on the parameter $t$ is called a co-extension of $\gamma$.

THEOREM 2.1. (See Theorem 5.2 of [4].) If all harmonic forms $\gamma \in \boldsymbol{H}^{r, s}\left(B_{0}\right)$ are extendible and co-extendible, then $\operatorname{dim} H_{\partial_{t}}^{r, s}\left(B_{t}\right)$ is constant in a neighborhood of $0 \in M$.

3. Harmonic theory. Let us consider the theory of harmonic forms for the holomorphic vector bundle $T_{0}^{\prime}$, with respect to the chosen hermitian metric on $V_{0}$. For a form $M \in \psi_{r, s}^{\prime}$ we have the orthogonal decomposition with respect to the inner product $(,)_{0}$

$$
M=\vartheta^{\prime} D^{\prime} G^{\prime} M+D^{\prime} \vartheta^{\prime} G^{\prime} M+H^{\prime} M
$$

where $D^{\prime}$ is given by $D^{\prime} M=\left[Q_{0}, M\right], \vartheta^{\prime}: \psi_{r, s+1}^{\prime} \rightarrow \psi_{r, s}^{\prime}$ is the adjoint of $D^{\prime}$ with respect to the product $(,)_{0}, G^{\prime}$ is the Green's operator $G^{\prime}: \psi_{r, s}^{\prime} \rightarrow \psi_{r, s}^{\prime}$ and $H^{\prime}: \psi_{r, s}^{\prime} \rightarrow H_{r, s}^{\prime}$ the harmonic projector. The conjugation operator

$$
S: T \otimes \mathscr{F}(r, s) \rightarrow T \otimes \mathscr{F}(s, r)
$$

associates to a form $a \otimes \alpha$ its conjugate $\bar{a} \otimes \bar{\alpha}$ and satisfies $S^{2}=I$; it is an antilinear bijection. Extending $S$ linearly to elements of $\psi$ and setting $S L=\bar{L}$ we get for $L \in \psi_{r, s}^{\prime}$, using the identities $I=P+Q$ and $[I, I]=0$,

$$
\overline{D^{\prime} L}=[\overline{Q, L}]=[\bar{Q}, \bar{L}]=-[P, \bar{L}]=-[Q, \bar{L}]=-D^{\prime \prime} \bar{L},
$$

where $D^{\prime \prime}$ is defined by $D^{\prime \prime} M=[Q, M]$ for $M \in \psi_{r, s}^{\prime \prime}$. Therefore

$$
S D^{\prime}=-D^{\prime \prime} S
$$

and $S$ induces an antilinear bijection of the cohomology groups

$$
S: H^{r, s}\left(\psi^{\prime}, D^{\prime}\right) \rightarrow H^{s, r}\left(\psi^{\prime \prime}, D^{\prime \prime}\right) .
$$

In particular $\operatorname{dim}_{C} H^{r, s}\left(\psi^{\prime}, D^{\prime}\right)=\operatorname{dim}_{C} H^{s, r}\left(\psi^{\prime \prime}, D^{\prime \prime}\right)$.

For $L, M \in \psi^{\prime}$, we have

$$
\left(\overline{\vartheta^{\prime} L}, \bar{M}\right)=\left(\bar{L}, \overline{D^{\prime} M}\right)=-\left(\bar{L}, D^{\prime \prime} \bar{M}\right) .
$$


For $N \in \psi^{\prime \prime}$, we can write

$$
\vartheta^{\prime \prime} N=-\overline{\vartheta^{\prime} \bar{N}}
$$

where $\vartheta^{\prime \prime}$ is the adjoint of $D^{\prime \prime}$ with respect to the product $($,$) . If we define the$ Green's operator $G^{\prime \prime}$ and the harmonic projector $H^{\prime \prime}$ for $N \in \psi^{\prime \prime}$ by

$$
G^{\prime \prime} N=\overline{G^{\prime} \bar{N}}, \quad H^{\prime \prime} N=\overline{H^{\prime} \bar{N}}
$$

we get for $N \in \psi^{\prime \prime}$ the orthogonal decomposition

$$
N=D^{\prime \prime} \vartheta^{\prime \prime} G^{\prime \prime} N+\vartheta^{\prime \prime} D^{\prime \prime} G^{\prime \prime} N+H^{\prime \prime} N .
$$

Define now the complex linear operations $D, G$, and $H$ on $\psi$ by their restrictions on $\psi^{\prime}$ and $\psi^{\prime \prime}: D\left|\psi^{\prime}=D^{\prime}, D\right| \psi^{\prime \prime}=D^{\prime \prime}$ etc. Note that $D L=[Q, L]$ for $L \in \psi$. We get the orthogonal decomposition for $N \in \psi$ :

$$
N=D \vartheta G N+\vartheta D G N+H N .
$$

4. The form $\xi_{t}$. Define a form $\xi_{t} \in \psi_{1}$ for each $t \in M$ by

$$
\xi_{t}=Q_{0}-Q_{t} .
$$

Proposition 4.1. The form $\xi_{t}$ satisfies the equation

$$
D \xi_{t}=\frac{1}{2}\left[\xi_{t}, \xi_{t}\right]
$$

for all $t \in M$.

Proof. The fact that the almost-complex structure associated with the projection $Q_{t}$ is integrable is expressed by the equation

$$
\left[Q_{t}, Q_{t}\right]=0
$$

(see $\left[1\right.$, p. 132]). Hence $0=\left[Q_{0}-\xi_{t}, Q_{0}-\xi_{t}\right]=-2\left[Q_{0}, \xi_{t}\right]+\left[\xi_{t}, \xi_{t}\right]$ which gives (4.2) as by definition $D \xi_{t}=\left[Q_{0}, \xi_{t}\right]$.

Let now for each $t \in M$ the projection $E_{t}$ in the complexified tangent bundle of $V_{0}$ be defined by

$$
\operatorname{Im} E_{t}=T_{0}^{\prime}, \quad \operatorname{Ker} E_{t}=T_{t}^{\prime \prime},
$$

where $T_{t}^{\prime}=\operatorname{Im} P_{t}=\operatorname{Ker} Q_{t}, T_{t}^{\prime \prime}=\operatorname{Ker} P_{t}=\operatorname{Im} Q_{t}$. Define for each $t \in M$ a form $\varphi_{t}$ by

$$
\varphi_{t}=E_{t} \pi Q_{0} .
$$

Obviously $\varphi_{t}$ is of type $(0,1)^{\prime}$ and $\varphi_{t}$ and $\xi_{t}$ depend differentiably on $t$. Furthermore $\varphi_{0}=\xi_{0}=0$.

From the definition of $\varphi_{t}$ we get

$$
\operatorname{Ker}\left(Q_{0}-\varphi_{t}\right)=T_{0}^{\prime}, \quad \operatorname{Im}\left(Q_{0}-\varphi_{t}\right)=T_{t}^{\prime \prime} .
$$

Therefore

$$
Q_{t}\left(Q_{0}-\varphi_{t}\right)=Q_{0}-\varphi_{t}, \quad Q_{t}\left(P_{0}-\bar{\varphi}_{t}\right)=0
$$


where $\bar{\varphi}_{t}$ denotes the conjugate of $\varphi_{t}$. By adding up we get

$$
Q_{t}\left(I-\varphi_{t}-\bar{\varphi}_{t}\right)=Q_{0}-\varphi_{t}
$$

As $\varphi_{t}(x) \rightarrow 0$ when $t \rightarrow 0$, uniformly on $V_{0}, I-\varphi_{t}-\bar{\varphi}_{t}$ has an inverse for small $t$. Suppose $\varepsilon$ is small enough so that this is true for all $t \in M=\left\{t \in \boldsymbol{R}^{m}|| t \mid<\varepsilon\right\}$. Then we can write

$$
Q_{t}=\left(Q_{0}-\varphi_{t}\right)\left(I-\varphi_{t}-\bar{\varphi}_{t}\right)^{-1} \text {. }
$$

Writing simply $\varphi$ for $\varphi_{t}$ we get from this

$$
Q_{t}=\left(Q_{0}-\varphi\right) \sum_{k=0}^{\infty}(\varphi+\bar{\varphi})^{k}=Q_{0}+\bar{\varphi} \sum_{k=0}^{\infty}(\varphi \bar{\varphi})^{k}(I+\varphi)-\sum_{k=0}^{\infty}(\bar{\varphi} \varphi)^{k}(I+\bar{\varphi}) .
$$

Set $\omega=\sum_{k=1}^{\infty}(\varphi \bar{\varphi})^{k}$. From the definition (4.1) of $\xi_{t}$ we get (writing $\xi$ for $\xi_{t}$ )

$$
\begin{aligned}
\pi_{1,0}^{\prime} \xi & =\omega, & & \pi_{0,1}^{\prime \prime} \xi=-\bar{\omega}, \\
\pi_{0,1}^{\prime} \xi & =\varphi+\varphi \bar{\omega}, & & \pi_{1,0}^{\prime \prime} \xi=-\bar{\varphi}-\bar{\varphi} \omega, \\
\xi & =\pi^{\prime} \xi-\overline{\pi^{\prime} \xi} . & &
\end{aligned}
$$

5. A development for the form $\xi$. The decomposition formula (3.6) together with (4.2) gives

$$
\xi=\vartheta G \frac{1}{2}[\xi, \xi]+h
$$

where $h=h_{t}=D \vartheta G \xi_{t}+H \xi_{t} \in Z\left(\psi_{1}\right)=\operatorname{Ker} D \mid \psi_{1}$. As there are (see [7, p. 146 and p. 150] and [9, p. 25]), for any nonnegative integer $k$, constants $c_{k}^{\prime}$ and $c_{k}^{\prime \prime}$ such that for any $\alpha, \beta$ in $\psi$

$$
\begin{gathered}
\|[\alpha, \beta]\|_{k} \leqq c_{k}^{\prime}\|\alpha\|_{k+1}\|\beta\|_{k+1}, \\
\|\vartheta G \alpha\|_{k+1} \leqq c_{k}^{\prime \prime}\|\alpha\|_{k},
\end{gathered}
$$

we get for $\alpha, \beta \in \psi_{1}$

$$
\left\|\vartheta G \frac{1}{2}[\alpha, \beta]\right\|_{k} \leqq c_{k}\|\alpha\|_{k}\|\beta\|_{k}
$$

with $c_{k}=\frac{1}{2} c_{k}^{\prime} c_{k}^{\prime \prime}$. Hence the quadratic function $Q$ defined by

$$
B \xi \xi=Q(\xi)=\vartheta G \frac{1}{2}[\xi, \xi]
$$

is continuous in the norm \|\|$_{k}$ from $\psi_{1}$ into itself. Thus it admits a continuous extension $\hat{Q}$ to the completion $\hat{\psi}_{1}$ of $\psi_{1}$ for the \|\|$_{k}$-norm. Setting

$$
F=I-\hat{Q}
$$

we can write the extension of (5.1) in the form

$$
F(\xi)=h
$$

where $F: \hat{\psi}_{1} \rightarrow \hat{\psi}_{1}$ is holomorphic in a neighborhood of $0 \in \hat{\psi}_{1}$ and $F^{\prime}(0)=I$. By 
the inverse function theorem $F^{-1}$ exists in a neighborhood of $0 \in \hat{\psi}_{1}$ and is holomorphic at the origin. Hence we have the unique development

$$
\xi=F^{-1} h=\sum_{i=1}^{\infty} A_{i} h^{i}, \quad h \in \psi_{1},
$$

valid in a \|\|$_{k}$-neighborhood of $0 \in \psi_{1}$, where $A_{i}$ for $i \geqq 1$ is an $i$-linear continuous map $\pi^{i} \psi_{1} \rightarrow \psi_{1}$ and $A_{1}=I$. Writing the formula (5.6) in the form

$$
\xi=h+\hat{B} \xi \xi
$$

we get by successive substitutions the recursive formula

$$
A_{n} h^{n}=\sum_{i+j=n} B A_{i} h^{i} A_{j} h^{j}
$$

for the coefficients of the development (5.8).

By the equation (4.2) $[\xi, \xi] \in D \psi_{1}$ or

$$
\sum_{n>2} \sum_{i+j=n}\left[A_{i} h^{i}, A_{j} h^{j}\right] \in D \psi_{1}
$$

for small $h$.

6. The differential equations. By the definition of the form $\xi_{t}$, the equation $\bar{\partial}_{t} \eta=0$, for $\eta \in L^{r, s}\left(B_{t}\right)$, is equivalent to

$$
\bar{\partial}_{0} \eta=\left[\xi_{t}, \eta\right]
$$

and the equation $\vartheta_{t} \sigma_{t}=0$, for $\sigma \in L^{r, s}\left(B_{t}\right)$, is equivalent to

$$
\bar{\partial}_{0} \#_{t} \sigma=\left[\xi_{t}, \#_{t} \sigma\right]
$$

where $\#_{t}$ is the anti-isomorphism,

$$
\#_{t}: B_{t} \otimes \mathscr{F}_{t}(r, s) \rightarrow B_{t}^{*} \otimes \mathscr{F}_{t}(n-r, n-s),
$$

defined by the hermitian metrics on $B_{t}$ and $V_{t}$ (see [3, p. 120]) and the bracket operation for $B$-valued forms is given by

$$
[N, \gamma]=D \gamma \pi N+(-1)^{n} D(\gamma \bar{\wedge} N), \quad N \in \psi, \gamma \in L(B),
$$

where $n=\operatorname{deg} N$. Then it follows that $\bar{\partial}_{t} \gamma=\left[Q_{t}, \gamma\right]$ for $\gamma \in L(B)$.

To investigate the space of solutions $\eta$ of the linear equation (6.1) we write

$$
\eta=\bar{\partial} \vartheta G \eta+\vartheta \bar{\partial} G \eta+H \eta
$$

where $\bar{\partial}$ stands for $\bar{\partial}_{0}$ etc. Substitution from (6.1) gives

$$
\eta=\vartheta G\left[\xi_{t}, \eta\right]+b
$$

where the "initial value" $b \in Z^{r, s}\left(B_{0}\right)$ corresponding to $\eta$ is given by

$$
b=\bar{\partial} \vartheta G \eta+H \eta \text {. }
$$

In fact, if $t=0$ the equation (6.6) gives $\eta=b$. 
It is clear that a solution of the "integral equation" (6.6) for a fixed $t$ is a solution of the differential equation (6.1) if and only if the integrability condition

$$
\left[\xi_{t}, \eta\right] \in \bar{\partial} L
$$

is satisfied.

7. Solution of the integral equation (6.6). There are positive constants (see $\left[9\right.$, p. 25]) $a_{k}^{\prime}$ and $a_{k}^{\prime \prime}$ such that

$$
\begin{gathered}
\|\vartheta G \gamma\|_{k} \leqq a_{k}^{\prime}\|\gamma\|_{k-1}, \quad \forall \gamma \in L^{r+s}(B), \\
\|[N, \gamma]\|_{k-1} \leqq a_{k}^{\prime \prime}\|N\|_{k}\|\gamma\|_{k}, \quad \forall N \in \psi_{1}, \quad \forall \gamma \in L^{r+s}(B) .
\end{gathered}
$$

For the linear map $f: L^{r+s}(B) \rightarrow L^{r+s}(B)$ defined by

$$
f \gamma=\gamma-\vartheta G\left[\xi_{t}, \gamma\right]
$$

we get, setting $a_{k}=a_{k}^{\prime} a_{k}^{\prime \prime}$,

$$
\left(1-a_{k}\left\|\xi_{t}\right\|_{k}\right)\|\eta\|_{k} \leqq\|f \eta\|_{k} \leqq\left(1+a_{k}\left\|\xi_{t}\right\|_{k}\right)\|\eta\|_{k} .
$$

Hence, for a fixed $t, f$ is continuous in the norm \|\|$_{k}$ and thus admits a continuous extension $f$ to the completion $L^{r+s}(B)$ of $L^{r+s}(B)$ with respect to the $k$-norm. By continuity the inequalities (7.4) hold for $\eta \in \hat{L}^{r+s}(B)$, and by the first of them $f$ is injective for $\left\|\xi_{t}\right\|_{k}<1 / a_{k}$; we assume $\varepsilon$ is small enough for this inequality to hold for all $t \in M=\left\{t \in R^{m}|| t \mid<\varepsilon\right\}$. If we set

$$
T_{t} \eta=\vartheta G\left[\xi_{t}, \eta\right], \quad \hat{f}=I-\hat{T}_{t},
$$

then $\left\|\hat{T}_{t}\right\|_{k}<1$ for $t \in M$, and thus $\hat{f}$ has a continuous inverse given by

$$
\eta_{t}=\hat{f}_{t}^{-1} b=b+\hat{T}_{t} b+\hat{T}_{t}^{2} b+\cdots, \quad b \in \hat{L}^{r+s}(B) .
$$

Substituting for $T_{t}$ from (7.5) and for $\xi_{t}$ from (5.9) and (5.10), we get for the solution $\eta_{t}$ of the integral equation (6.6) with the initial value $b_{t} \in \boldsymbol{H}^{r, s}\left(B_{0}\right)$ the expression

$$
\eta_{t}=b_{t}+\sum_{n \geqq 1} \vartheta G P_{n}\left(h_{t}^{n}, b\right)
$$

where

$$
P_{n}\left(h_{t}^{n}, b\right)=\sum_{1 \leqq k \leqq n} \sum_{i_{1}+\cdots+i_{k}=n}\left[A_{i_{1}} h_{t^{1}}^{i_{1}}, \vartheta G\left[A_{i_{2}} h^{i_{2}}, \ldots, \vartheta G\left[A_{i_{k}} h^{i_{k}}, b_{t}\right] \ldots\right]\right] .
$$

For $b_{t} \in \boldsymbol{H}^{r, s}\left(B_{0}\right)$ we have $\eta_{t} \in L^{r+s}\left(B_{0}\right)$, and if $b_{t}$ depends differentiably on $t$ so does $\eta_{t}$ (see [7, p. 150]). Using the development (5.8) for $\xi_{t}$ we get

$$
\left[\xi_{t}, \eta_{t}\right]=\sum_{n \geqq 1} P_{n}\left(h^{n}, b_{t}\right)
$$

The function $h \rightarrow P_{n}\left(h^{n}, b\right)$ is $n$-homogeneous and continuous in the norm \|\|$_{k}$ from $\psi_{1}$ into $L^{r+s+1}(B)$. 
8. The integrability condition. Consider now the integrability condition (6.8). By (7.9) it can be written in the form

$$
\sum_{n \geqq 1} P_{n}\left(h_{t}^{n}, b\right) \in \bar{\partial} L, \quad b \in Z^{r, s}\left(L\left(B_{0}\right)\right) .
$$

To analyse this condition we need the following result.

LEMMA 8.1. Let $Y$ be a closed vector subspace of a Banach space $X, Z$ another Banach space. For each positive integer $k$, let $L_{k}$ be a $k$-linear continuous function

$$
L_{k}: Z^{k} \rightarrow X
$$

such that

$$
S(h)=\sum_{k \geqq 1} L_{k} h^{k}
$$

converges for $|h|<\varepsilon$. Then, for a fixed $h \in Z$ with $|h|<\varepsilon, S(t h) \in Y$ for $-1 \leqq t \leqq 1$ if and only if $L_{k} h^{k} \in Y$ for all $k \geqq 1$.

Proof. The sufficiency of the condition is evident. Suppose then that $S(t h) \in Y$ for a fixed $h,|h|<\varepsilon$ and for $-1 \leqq t \leqq 1$. Then if $f(t)=S(t h), f:[-1,1] \rightarrow Y$ is differentiable, indeed analytic at $0 \in R$, as the composition of two analytic functions. Differentiating term by term the development $f(t)=\sum_{k \geqq 1} t^{k} L_{k} h^{k}$ we get

$$
f^{(n)}(t)=\sum_{k \geqq n} k(k-1) \cdots(k-n+1) t^{k-n} L_{k} h^{k},
$$

from which

$$
f(0)=0, \quad f^{(n)}(0)=n ! L_{n} h^{n} .
$$

But, as $Y$ is closed in $X, f(t) \in Y$ for $\forall t$ implies $f^{(n)}(0) \in Y$ for $\forall n$. Therefore $L_{k} h^{k} \in Y$ for $k \geqq 1$, as was to be shown.

As $\bar{\partial} L(B)$ is a closed subspace of $L(B)$ for the norm \|\|$_{k}$, the condition

$$
P_{n}\left(h_{t}^{n}, b\right) \in \bar{\partial} L(B), \quad \forall n \geqq 1,
$$

is sufficient for $b \in \boldsymbol{H}^{r, s}\left(B_{0}\right)$ to be integrable at $t$ (for $f^{-1}(b)$ to be a solution of (6.1)).

Therefore we have the following:

THEOREM 8.1. Let $b \in \boldsymbol{H}^{r, s}\left(B_{0}\right)$. In order that $f_{t}^{-1}(b)=\eta_{t}$ is a solution of $(6.1)$ for each $t \in M$, it is sufficient that

$$
P_{n}\left(h_{t}, b\right) \in \bar{\partial} L(B), \quad \forall t \in M, \quad n \geqq 1 .
$$

The solution $\eta_{t}$ is a $\bar{\partial}_{t}$-closed B-form of type $(r, s)$, and it depends differentiably on $t$.

Let now $A_{\varepsilon}$ be the balanced hull of $\left\{h_{t}|| t \mid<\varepsilon\right\}$ in $Z\left(\psi_{1}\right)$, i.e. $A_{\varepsilon}=\left\{s h_{t}|| t \mid<\varepsilon\right.$ and $-1 \leqq s \leqq 1\}$. 
THEOREM 8.2. The condition

$$
P_{n}(h, b) \in \bar{\partial} L(B), \quad \forall h \in A_{\varepsilon}, \quad \forall n \geqq 1,
$$

implies (8.2). In case $\left\{h_{t}|| t \mid<\varepsilon\right\}=A_{\varepsilon}$, the condition (8.3) is necessary and sufficient for $f_{t}^{-1}(b)=\eta_{t}$ to be a solution of (6.1) for each $t \in M$.

The last statement of the theorem follows from the Lemma 8.1.

9. Conditions for $\operatorname{dim} H_{\bar{\partial}_{t}}^{r, s}\left(B_{t}\right)$ to be locally constant. A form $\gamma \in \bar{\partial} L^{r, s-1}\left(B_{0}\right)$ is trivially extendible; it is sufficient to take $\eta_{t}=\pi_{r, s}(t) \vec{\partial}_{t} \sigma$ as an extension, if $\gamma=\bar{\partial} \sigma$. Therefore it is no restriction to limit oneself to harmonic initial values $b$ in the following theorem which follows from Theorem 8.2.

THEOREM 9.1. In order that all $\bar{\partial}$-closed forms $\gamma \in L^{r, s}\left(B_{0}\right)$ are extendible with any initial value $b_{t} \in Z^{r, s}\left(L\left(B_{0}\right)\right)$ at $t=0$, it is sufficient that (8.3) holds for $\forall h \in A_{\varepsilon}$ and $\forall b \in \boldsymbol{H}^{r, s}\left(B_{0}\right)$.

By Theorem $2.1 \operatorname{dim} H \frac{r, s}{\partial_{t}}\left(B_{t}\right)$ is constant in a neighborhood of $t=0$ if all harmonic forms $\gamma \in \boldsymbol{H}^{r, s}\left(B_{0}\right)$ are extendible and co-extendible. Therefore we get the following result. Let $\mathscr{R}(\bar{\partial})$ stand for $\bar{\partial}\left(L\left(B_{0}\right)\right)$ or $\bar{\partial}\left(L\left(B_{0}^{*}\right)\right)$ depending on the case.

THEOREM 9.2. In order that $\operatorname{dim} H_{\dot{\partial}_{t}}^{r . s}\left(B_{t}\right)$ is constant in a neighborhood of $t=0$, it is sufficient that there exists $\varepsilon>0$ such that, for all $h \in A_{\varepsilon}$ and all $n \geqq 1$,

$$
P_{n}(h, b) \in \mathscr{R}(\bar{\partial})
$$

whenever $b \in \boldsymbol{H}^{r, s}\left(B_{0}\right)$ or $b \in \boldsymbol{H}^{n-r, n-s}\left(B_{0}^{*}\right)$. The condition is also necessary if $h(M)$ is a cone centered at $0 \in Z\left(\psi_{1}\right)$.

To obtain necessary conditions in the general case, suppose that all forms $\gamma \in \boldsymbol{H}^{r, s}\left(B_{0}\right)$ are extendible. Suppose $t \rightarrow b_{t}$ is the initial value function of an extension $\gamma_{t}$ of $\gamma$. Then

$$
\gamma_{t}=\sum_{n \geqq 1} P_{n}\left(h_{t}^{n}, b_{t}\right) \in \bar{\partial} L\left(B_{0}\right)
$$

As $t \rightarrow h_{t}$ is differentiable as a function from $M \subset R^{m}$ into the Banach space $\hat{\psi}_{1}$, and the series (9.3) and the series of the derivatives are uniformly convergent for $|t| \leqq \delta<\varepsilon$ with $\delta$ sufficiently small, we can differentiate term by term. This gives for $\Delta \in \boldsymbol{R}^{m}$

$$
\gamma_{i}^{\prime} \Delta=\sum_{n \geqq 1}\left\{n P_{n}\left(h_{t}^{n-1} h_{t}^{\prime} \Delta, b_{t}\right)+P_{n}\left(h_{t}^{n}, b_{t}^{\prime} \Delta\right)\right\} \in \bar{\partial} L\left(B_{0}\right),
$$

where $h_{t}^{\prime}$ and $b_{t}^{\prime}$ are derivatives of $h_{t}$ and $b_{t}$, as functions from $M$ into $\hat{\psi}_{1}$ and $\hat{L}^{r, s}\left(B_{0}\right)$, respectively.

For $t=0$ we have, as $h_{0}=0$,

$$
R_{1}\left(\Delta_{1}, b_{0}\right)=P_{1}\left(h_{0}^{\prime} \Delta_{1}, b_{0}\right) \in \bar{\partial} L\left(B_{0}\right), \quad \forall \Delta_{1} \in R^{m},
$$


with

$$
R_{1}\left(\Delta_{1}, b_{0}\right)=\left[h_{0}^{\prime} \Delta_{1}, b_{0}\right] .
$$

Differentiating the expression (9.4) gives

$$
\begin{aligned}
& \gamma_{t}^{\prime \prime} \Delta_{2} \Delta_{1}=\sum_{n \geqq 1}\{ n(n-1) P_{n}\left(h_{t}^{n-2} h_{t}^{\prime} \Delta_{2} h_{t}^{\prime} \Delta_{1}, b_{t}\right) \\
&+n P_{n}\left(h_{t}^{n-1} h_{t}^{\prime \prime} \Delta_{2} \Delta_{1}, b_{t}\right)+n P_{n}\left(h_{t}^{n-1} h_{t}^{\prime} \Delta_{1}, b_{t}^{\prime} \Delta_{2}\right) \\
&\left.+n P_{n}\left(h_{t}^{n-1} h_{t}^{\prime} \Delta_{2}, b_{t}^{\prime} \Delta_{1}\right)+P_{n}\left(h_{t}^{n}, b_{t}^{\prime \prime} \Delta_{2} \Delta_{1}\right)\right\} \in \bar{\partial} L\left(B_{0}\right) .
\end{aligned}
$$

This is permitted as once more the series of derivatives converges uniformly with the series (9.4). Setting $t=0$ one obtains

$$
\begin{aligned}
2 P_{2}\left(h_{0}^{\prime} \Delta_{2} h_{0}^{\prime} \Delta_{1}, b_{0}\right) & +P_{1}\left(h_{0}^{\prime \prime} \Delta_{2} \Delta_{1}, b_{0}\right)+P_{1}\left(h_{0}^{\prime} \Delta_{1}, b_{0}^{\prime} \Delta_{2}\right) \\
& +P_{1}\left(h_{0}^{\prime} \Delta_{2}, b_{0}^{\prime} \Delta_{1}\right) \in \bar{\partial} L\left(B_{0}\right) .
\end{aligned}
$$

By (9.5) the last two terms can be written in the form $R_{1}\left(\Delta_{1}, b_{0}^{\prime} \Delta_{2}\right)-R_{1}\left(\Delta_{2}, b_{0}^{\prime} \Delta_{1}\right)$ and are thus in $\bar{\partial} L\left(B_{0}\right)$. Setting

$$
R_{2}\left(\Delta_{2} \Delta_{1}, b_{0}\right)=2 P_{2}\left(h_{0}^{\prime} \Delta_{2} h_{0}^{\prime} \Delta_{1}, b_{0}\right)+P_{1}\left(h_{0}^{\prime \prime} \Delta_{2} \Delta_{1}, b_{0}\right)
$$

we get therefore from $(9.8)$

$$
R_{2}\left(\Delta_{2} \Delta_{1}, b_{0}\right) \in \bar{\partial} L\left(B_{0}\right), \quad \Delta_{2}, \Delta_{1} \in \boldsymbol{R}^{m} .
$$

Generally one obtains

$$
\begin{aligned}
R_{k}\left(\Delta_{k} \Delta_{k-1} \cdots \Delta_{1}, b_{0}\right)= & {\left[\left(D_{t}\right)^{k} \sum_{i=1}^{k} P_{i}\left(h_{t}^{i}, b_{0}\right)\right]_{t=0} \Delta_{k} \cdots \Delta_{1} } \\
= & k ! P_{k}\left(h_{0}^{\prime} \Delta_{k} \cdots h_{0}^{\prime} \Delta_{1}, b_{0}\right) \\
& +\cdots+P_{1}\left(h_{0}^{(k)} \Delta_{k} \cdots \Delta_{1}, b_{0}\right) \in \bar{\partial} L,
\end{aligned}
$$

the other terms in $\left[\left(D_{t}\right)^{k} \gamma_{t}\right]_{t=0} \Delta_{k} \cdots \Delta_{1}$ being expressible in terms of $R_{k-1}, \ldots, R_{1}$, and hence in $\bar{\partial} L\left(B_{0}\right)$. Hence we have the

THEOREM 9.3. The conditions

$$
R_{k}\left(\Delta_{k} \cdots \Delta_{1}, b\right) \in \mathscr{R}(\bar{\partial})
$$

for $\forall \Delta_{i} \in \boldsymbol{R}^{m}$ and $\forall b \in \boldsymbol{H}^{r, s}\left(B_{0}\right), \forall b \in \boldsymbol{H}^{n-r, n-s}\left(B_{0}^{*}\right)$ are necessary for $\operatorname{dim} H_{\partial_{t}}^{r, s}\left(B_{t}\right)$ to be constant in a neighborhood of $t=0$. If the family is real analytic, then the conditions are also sufficient.

For the last statement, let us note that if the family is real analytic, then we can write $h_{t}=\sum_{k \geqq 1}(1 / k !) h_{0}^{(k)} t^{k}$ where the series converges in a neighborhood of $t=0$. This implies the convergence of

$$
\eta_{t}=\sum_{k \geqq 1} \frac{1}{k !} R_{k}\left(t^{k}, b_{0}\right)+b_{0},
$$


for small $t$. Furthermore, $\gamma_{t}=\sum_{n \geqq 1} P_{n}\left(h_{t}^{n}, b_{0}\right)$ is analytic in $t$ in a neighborhood of $t=0$ and, by the definition (9.11) of $R^{k}$, we have $\gamma_{t}=\eta_{t}$ for small $t$. Therefore $\eta_{t}$ is a solution of the integral equation (6.6) with a constant initial value function $b_{0}$, and the conditions (9.12) imply that the integrability condition (6.8) is satisfied. As $\eta_{0}=b_{0} \in \boldsymbol{H}^{r, s}\left(B_{0}\right)$ is arbitrary, it follows that all forms in $\boldsymbol{H}^{r, s}\left(B_{0}\right)$ are extendible (analogously for co-extendibility). The local constancy of $\operatorname{dim} H_{\bar{\partial}_{t}}^{r, s}\left(B_{t}\right)$ follows then by the Theorem 2.1 .

\title{
REFERENCES
}

1. A. Froehlicher, E. Kobayashi and A. Nijenhuis, Deformation theory of complex manifolds, Lecture Notes, University of Washington, Seattle, Wash., 1957.

2. Ph. A. Griffiths, The extension problem for compact submanifolds of complex manifolds. I. The case of a trivial normal bundle, Proc. Conference Complex Analysis (Minneapolis, 1964), Springer, Berlin, 1965, pp. 113-142. MR 32 \#8362.

3. F. Hirzebruch, Topological methods in algebraic geometry, 3rd ed., Die Grundlehren der math. Wissenschaften, Band 131, Springer-Verlag, New York, 1966. MR 34 \#2573.

4. T. Klemola, Transportable forms, Canad. Math. Bull. (to appear).

5. K. Kodaira and D. C. Spencer, On deformations of complex analytic structures. I, II, Ann. of Math. (2) 67 (1958), 328-466. MR 22 \#3009.

6. - On deformations of complex analytic structures. III, Ann. of Math. (2) 71 (1960), 43-76. MR 22 \#5991.

7. M. Kuranishi, New proof for the existence of locally complete families of complex structures, Proc. Conference Complex Analysis (Minneapolis, 1964), Springer, Berlin, 1965, pp. 142-154. MR 31 \#768.

8. H. K. Nickerson, On differential operators and connections, Trans. Amer. Math. Soc. 99 (1961), 509-539. MR 28 \#5411.

9. L. Nirenberg, Partial differential equations with applications in geometry, Lectures on Modern Math., vol. 2, Wiley, New York, 1964. MR 31 \#2477.

\author{
UNIVERSITY OF MONTREAL, \\ Montreal, Quebec, Canada
}

